\title{
Comparison of the intra-articular treatment with a platelet concentrate, high-density hyaluronan, triamcinolone hexacetonide, and stanozolol in dogs with hip osteoarthritis
}

\author{
Alves J. ${ }^{1,2, ~ *}$; Santos A. '; Jorge P. ' ; Lavrador C. ${ }^{2}$; Carreira L. Miguel3,4
}

Guarda Nacional Republicana; 'bIMED - Mediterranean Institute for Agriculture, Environment and Development, Universidade de Évora; ${ }^{3}$ Interdisciplinary Centre for Research in Animal Health (CIISA) - University of Lisbon, (FMV/ULisboa) - Portugal. ${ }^{4}$ Anjos of Assis Veterinary Medicine Centre; *alves.jca@gnr.pt

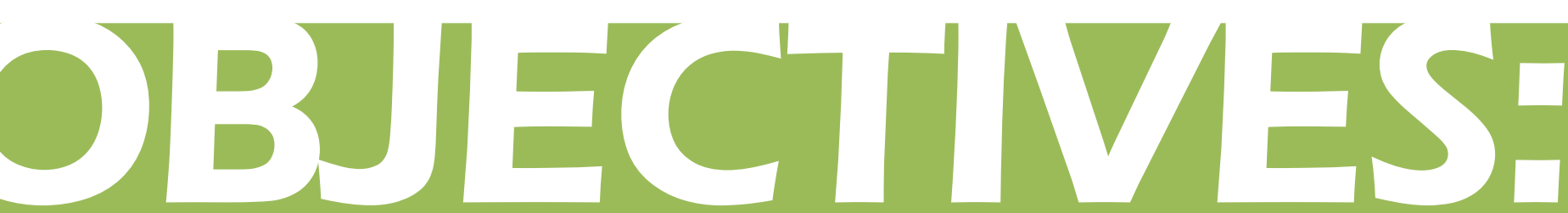

To compare the effect of the intra-articular treatment with triamcinolone hexacetonide (TH), stanozolol, hyaluronan, and platelet concentrate (PC) in Police working dogs with bilateral hip osteoarthritis (OA).

\section{MATERIALS AND METHODS:}

Fifty ( $N=50)$ police working dogs with hip $O A$ were randomly assigned to a control group (CG, $n=10$ ), TH group (THG, $n=10$ ), PC group (PCG, $\mathrm{n}=10$ ), stanozolol group (SG, $\mathrm{n}=10$ ) and Hylan $\mathrm{G}-\mathrm{F} 20$ group (HG);

On days $0,8,15,30,90$, and 180 days post-treatment, weight-bearing distribution, and the Canine Brief Pain Inventory, Liverpool Osteoarthritis in Dogs, and Canine Orthopedic Index) were evaluated.

Kaplan-Meier estimators were conducted and compared with the logrank test. Cox proportional hazard regression analysis was performed to determine treatment survival, $p<0.05$.

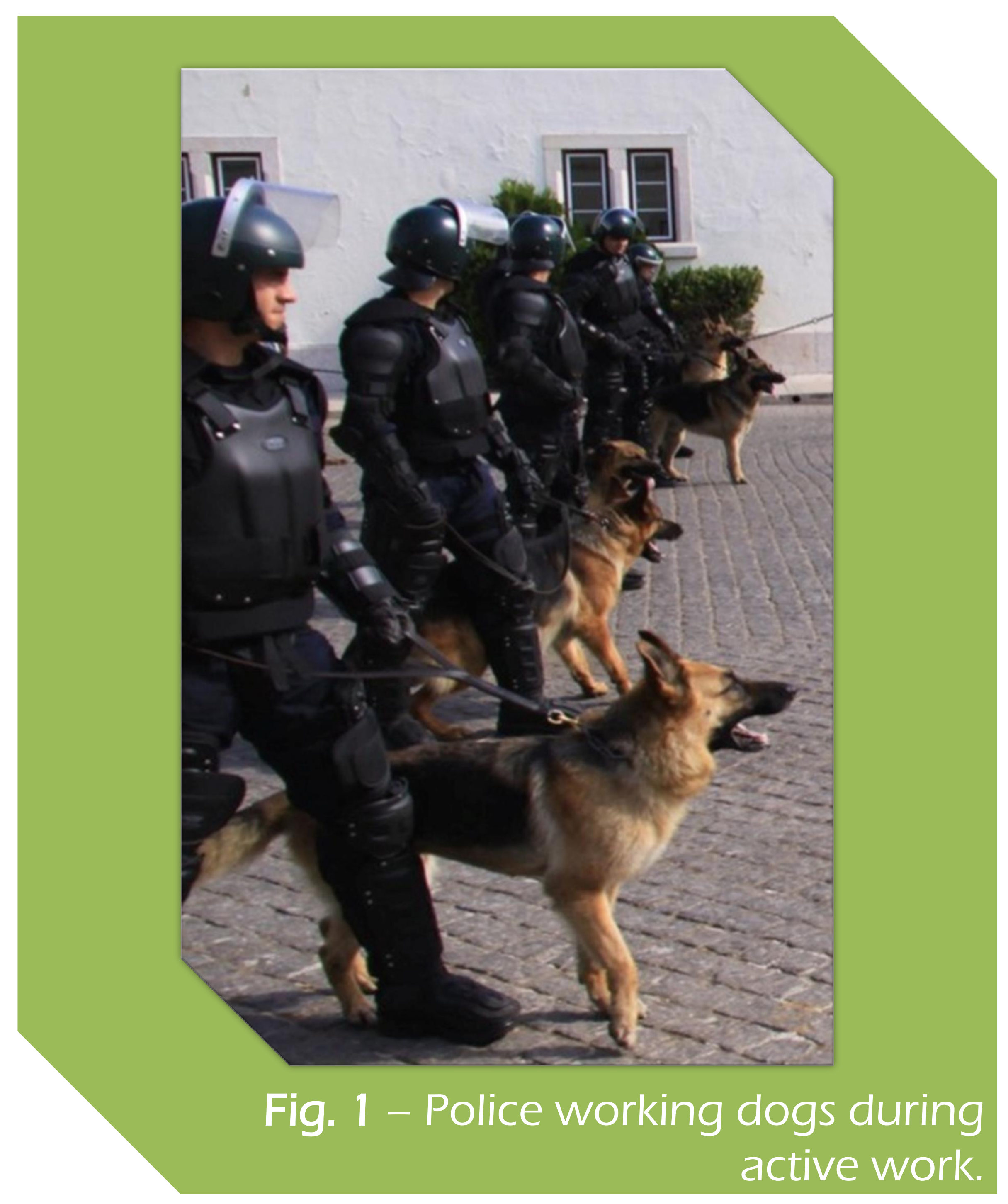

\section{RESULTS:}

Patients had a mean age of $6.5 \pm 2.4$ years and bodyweight of $26.7 \pm 5.2 \mathrm{~kg}$. At initial evaluation, hips were graded as mild $(n=35)$, moderate $(n=10)$, and severe $(N=5)$, and no differences were found between groups.

All treatments were able to improve CMI scores and weight-bearing,

PCG showed a lower range of variation while maintaining a positive result for more extended periods.

Breed, age, sex, and OFA grade did not significantly influence response to treatment.

Results are shown in the table.

\begin{tabular}{|c|c|c|c|c|c|c|c|c|}
\hline \multirow{2}{*}{ Variable } & \multicolumn{2}{|c|}{$\begin{array}{c}\text { Wheight distribution } \\
\text { Simmetry Index }(\mathrm{p}=0.014)\end{array}$} & \multicolumn{2}{|c|}{$\begin{array}{c}\text { CBPI } \\
\text { PIS }(\mathrm{p}=0.025)\end{array}$} & \multicolumn{2}{|c|}{$\begin{array}{c}\text { LOAD } \\
(\mathrm{p}=0.006)\end{array}$} & \multicolumn{2}{|c|}{$\begin{array}{c}\text { COI } \\
\text { Total }(\mathrm{p}=\mathbf{0 . 0 5 3})\end{array}$} \\
\hline & HR $(95 \%$ CI $)$ & $\mathbf{p}$ & HR $(95 \%$ CI $)$ & p & HR $(95 \%$ CI $)$ & p & HR $(95 \%$ CI $)$ & $\mathbf{p}$ \\
\hline Treatment & 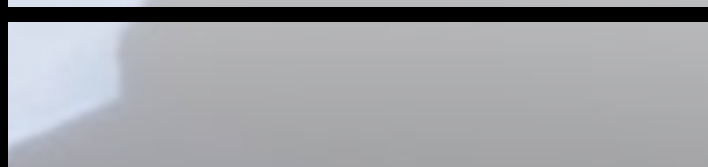 & $0,001 *$ & & $\overline{0,001 *}$ & & $\overline{0,000^{*}}$ & & 0,163 \\
\hline Control & 1,00 & & 1,00 & & 1,00 & & 1,00 & \\
\hline $\mathrm{HG}$ & $0,23(0,08-0,65)$ & $0,006^{*}$ & $0,06(0,15-0,26)$ & $0,000 *$ & $0,06(0,02-0,25)$ & $0,000^{*}$ & $0,29(0,09-0,92)$ & $0,036^{*}$ \\
\hline PCG & $0,24(0,21-0,26)$ & $0,000^{*}$ & $0,12(0,03-0,43)$ & $0,001^{*}$ & $0,19(0,06-0,62)$ & $0,005^{*}$ & $0,29(0,09-0,87)$ & $0,030^{*}$ \\
\hline SG & $0,21(0,07-0,60)$ & $0,004^{*}$ & $0,09(0,02-0,36)$ & $0,001^{*}$ & $0,08(0,02-0,28)$ & $0,000^{*}$ & $0,54(0,17-1,66)$ & 0,279 \\
\hline THG & $0,29(0,09-0,83)$ & $0,021^{*}$ & $0,08(0,02-0,32)$ & $0,000 *$ & $0,09(0,03-0,36)$ & $0,001^{*}$ & $0,49(0,17-1,41)$ & 0,188 \\
\hline OFA score & & 0,582 & & 0,621 & & 0,211 & & $0,041^{*}$ \\
\hline Mild & 1,00 & & 1,00 & & 1,00 & & 1,00 & \\
\hline Moderate & $0,70(0,29-1,69)$ & 0,430 & $1,53(0,65-3,61)$ & 0,330 & $2,18(0,89-5,34)$ & 0,088 & $0,69(0,28-1,73)$ & 0,431 \\
\hline Severe & $0,58(0,17-1,94)$ & 0,378 & $1,25(0,31-5,00)$ & 0,749 & $1,03(0,23-4,61)$ & 0,972 & $4,19(1,08-16,24)$ & $0,038^{*}$ \\
\hline Breed & & 0,861 & & 0,631 & & 0,073 & & 0,997 \\
\hline $\mathrm{LR}$ & 1,00 & & 1,00 & & 1,00 & & 1,00 & \\
\hline GSD & $0,74(0,24-2,28)$ & 0,589 & $0,52(0,14-1,92)$ & 0,327 & $0,76(0,21-2,76)$ & 0,672 & $0,92(0,26-3,28)$ & ${ }_{0,898}$ \\
\hline $\mathrm{BM}$ & $0,63(0,21-1,91)$ & 0,411 & $0,46(0,14-1,58)$ & 0,217 & $0,35(0,09-1,33)$ & 0,121 & $0,92(0,29-2,93)$ & ${ }_{0,893}$ \\
\hline DSD & $0,79(0,22-2,86)$ & 0,724 & $0,43(0,10-1,82)$ & 0,249 & $0,17(0,03-0,83)$ & $0,028^{*}$ & $1,00(0,24-4,15)$ & 0,995 \\
\hline & & & & & & & & \\
\hline $\begin{array}{l}\text { Male } \\
\text { Female }\end{array}$ & $\begin{array}{c}1,00 \\
151(076-209)\end{array}$ & & $\begin{array}{c}1,00 \\
085(037-107)\end{array}$ & 0704 & $\begin{array}{c}1,00 \\
117(040-279)\end{array}$ & 0721 & $\begin{array}{c}1,00 \\
200(09-455)\end{array}$ & 5080 \\
\hline Age & $0,90(0,77-1,06)$ & $\begin{array}{l}0,250 \\
0,216\end{array}$ & $0,912(0,77-1,08)$ & 0,299 & $0,9(0,76-1,07)$ & 0,229 & $\begin{array}{l}1,14(0,94-1,37) \\
1,0,(0,0)-4, J J)\end{array}$ & 0,180 \\
\hline
\end{tabular}

